\title{
Análise Comparativa de Testes Diagnnósticos para Diabete Gestacional
}

\author{
Comparison of Diagnostic Tests for Gestational Diabetes \\ Leila Katz, Melania Amorim, Isabela Coutinho, Luiz Carlos Santos
}

\section{RESUM0}

\begin{abstract}
Objetivos: avaliar os resultados maternos e perinatais de pacientes submetidas à curva glicêmica com $100 \mathrm{~g}$ de glicose, de acordo com três diferentes critérios diagnósticos.

Métodos: realizou-se estudo do tipo corte transversal, incluindo 210 pacientes assistidas no Instituto Materno-Infantil de Pernambuco (IMIP), submetidas durante a gravidez ao teste oral de tolerância à glicose $100 \mathrm{~g}$ (TOTG), com gestação única, sem história de diabete ou intolerância aos carboidratos prévia à gestação e cujo parto foi assistido no IMIP. Estas foram classificadas nos grupos: controles, pacientes com hiperglicemia leve, diabete gestacional (DG) de acordo com os critérios de Bertini, de Carpenter e Coustan e do "National Diabetes Data Group" (NDDG). Analisaram-se esses grupos, buscando-se associação entre a classificação das pacientes nos grupos e a presença de pré-eclâmpsia, recém-nascidos grandes para a idade gestacional (GIG) e freqüência de cesarianas e natimortos, comparando-se ainda as médias de peso ao nascer.

Resultados: a freqüência de DG de acordo com os critérios de Bertini, de Carpenter e Coustan e do NDDG foi de 48,1, 18,1, e 9\%, respectivamente, ao passo que a freqüencia de hiperglicemia leve foi de 10,5\%. A idade das pacientes aumentou progressivamente de acordo com o maior grau de intolerância aos carboidratos. Os grupos não diferiram quanto à freqüência de GIG, cesarianas, natimortos e médias de peso ao nascer. Verificou-se aumento significativo da incidência de pré-eclâmpsia em pacientes com hiperglicemia e DG por Carpenter e Coustan, mas não nos outros grupos.

Conclusões: a prevalência de diabete gestacional encontrada variou entre 9 e 48\%, de acordo com os diversos critérios, mas não se observaram diferenças significativas nos resultados maternos e perinatais entre os grupos. Critérios muito rígidos de diagnóstico podem levar a diagnóstico excessivo, sem melhora subseqüente do prognóstico perinatal.
\end{abstract}

PALAVRAS-CHAVE: Diabete gestacional. Hipertensão. Óbito fetal. Teste oral de tolerância à glicose.

\section{Introdução}

O diabete melito representa a complicação médica mais comum na gravidez. Segundo a classificação do "Report of the Expert Committee on the Diagnosis and Classification of Diabetes mellitus" (1997) ${ }^{1}$, diabete melito gestacional (DG) é definido como qualquer grau de intolerância à glicose com início ou primeiro reconhecimento

Instituto Materno-Infantil de Pernambuco (IMIP); Centro de Atenção à Mulher (CAM)

Correspondência:

Melania Maria Ramos de Amorim

Rua dos Coelhos, 300 - Boa Vista

50.070-550 - Recife - PE

e-mail: melamorim@uol.com.br durante a gestação.

A prevalência de DG varia bastante, dependendo da população estudada. Nos Estados Unidos da América, essa entidade complica em torno de 2-4\% das gestações, ao passo que representa em Recife ocorrem aproximadamente 135.000 casos anualmente. No (IMIP), sua prevalência é de $5 \%{ }^{2}$.

Além da influência racial implicando prevalências tão discordantes, temos que levar em consideração as diferentes rotinas adotadas em cada local para o rastreio de DG e dos diferentes critérios utilizados para o seu diagnóstico. Solomon et al. ${ }^{3}$ utilizando o coorte do "Nurse's Health Study II", avaliaram o rastreio e o diagnóstico, que foram insatisfatórios (fugindo às recomendações mais freqüentes) mesmo nesta popu- 
lação altamente selecionada. As diferentes condutas em relação a quem se vai rastrear e que nível será considerado como anormal alteram sobremaneira a prevalência da doença.

O diagnóstico do DG tem sido tema de intenso debate. Várias propostas foram feitas e colocadas em prática sem, no entanto, existir um consenso em relação ao melhor método. Os primeiros autores a apresentar um teste diagnóstico foram O'Sullivan \& Mahan ${ }^{4}$, em 1964. Os autores consideraram os melhores pontos de corte para predizer diabete no futuro, propondo-os como valores máximos de normalidade para o teste oral de tolerância à glicose (TOTG) na gestação. O trabalho dos autores foi pioneiro, constituindo, sem dúvida, uma das maiores contribuições na história do DG.

Nos anos seguintes, houve modificação das técnicas diagnósticas utilizadas e a glicemia deixou de ser medida no sangue total e passou a ser utilizado o plasma venoso. O "National Diabetes Data Group (NDDG)”, em 1979, modificou os valores propostos por O'Sullivan para compensar essas mudanças. Esses critérios são até hoje muito utilizados, tendo inclusive a recomendação do Colégio Americano de Ginecologistas e Obstetras. Em 1982, Carpenter e Coustan ${ }^{6}$ propuseram nova mudança nos valores preconizados para avaliação do TOTG, devido às modificações da técnica utilizada para determinação de glicose no plasma, uma vez que no lugar da técnica de Somogyi-Nelson, passou-se a usar o método enzimático. A nova curva proposta derivou dos valores originais de O'Sullivan após correções feitas devido a essa modificação.

Apesar de ser um divisor de águas no diagnóstico do DG, o estudo de O'Sullivan e Mahan, ao ser analisado do ponto de vista metodológico, apresenta falhas, principalmente no que diz respeito à derivação das conclusões e da validação do exame como técnica diagnóstica, o que foi demonstrado de forma clara por Naylor ${ }^{7}$, em 1989. Uma das questões levantadas é que os estudiosos tinham como objetivo predizer o desenvolvimento de diabete melito em longo prazo, levando em consideração essa característica, para selecionar os pontos de corte. O mais adequado teria sido procurar registrar as complicações neonatais imediatas e em longo prazo e correlacionálas com os valores do TOTG. Outro ponto importante é que a dicotomia entre normal/anormal é difícil de ser estabelecida e que provavelmente uma estratificação de acordo com níveis glicêmicos encontrados seria uma forma mais racional de se calcularem os riscos. Apesar de todas essas críticas, reconhece-se que, infelizmente, os critérios do NDDG estão tão estabelecidos como padrão para o diagnóstico de DG que pode ser difícil, ética e legalmente, iniciar os estudos necessários para se quantificarem riscos e definir estratégias de tratamento ${ }^{7}$.

Em 1998, realizou-se conferência internacional sobre diabete gestacional em Chicago, tendo a participação de um grande número de estudiosos de DG. Os participantes recomendaram o uso dos critérios propostos por Carpenter e Coustan para a avaliação do TOTG com 100 g de glicose ${ }^{8}$.

Os critérios diagnósticos ideais para DG ainda constituem tema de debate. Existem recomendações de vários órgãos, associações e estudiosos, e cada serviço adota aquela que lhe parece mais adequada. A multiplicidade de critérios diagnósticos dificulta a uniformização do tratamento ministrado às diabéticas gestacionais de forma mundial e prejudica as pesquisas feitas sobre o assunto, porque muitas vezes essas não podem ser comparadas. Há necessidade de realização de estudos demonstrando qual o melhor critério diagnóstico. Nesse sentido, o presente estudo teve como objetivo determinar a prevalência de DG (de acordo com os diversos critérios de interpretação) e de hiperglicemia leve entre as pacientes que realizaram curva glicêmica durante o pré-natal no IMIP, descrevendo as características maternas e perinatais de cada um dos grupos.

\section{Métodos}

O estudo foi desenvolvido no IMIP, Recife/ PE. Realizou-se um estudo do tipo corte transversal, analisando as características maternas, da gestação, parto e do recém-nascido de gestantes que realizaram curva glicêmica no CAM-IMIP, no período de 20/10/1999 a 22/11/2000. Pesquisaram-se os livros de registro de exames do setor de bioquímica do laboratório do IMIP, identificandose todas as pacientes que realizaram curva glicêmica após administração de $100 \mathrm{~g}$ de glicose no período do estudo. A partir dessa listagem fezse, então, busca dos prontuários no arquivo do serviço. Foram incluídas no estudo somente as pacientes com gestação tópica e única, tendo realizado o exame durante o acompanhamento prénatal no IMIP no segundo ou terceiro trimestres da gestação, com o parto assistido nesta instituição. Foram excluídas do estudo pacientes com o diagnóstico de diabete melito ou intolerância prévia aos carboidratos.

Após interpretação da curva glicêmica, as pacientes foram classificadas em controles (quatro pontos da curva glicêmica, normais por todas as classificações), hiperglicemia leve (um valor alterado da curva glicêmica de acordo com a classificação de Carpenter e Coustan ${ }^{9}$ ), diabete gesta- 
cional por Bertini (dois ou mais valores da curva alterados de acordo com a classificação de BertiniOliveira \& Camano $^{10}$ ), diabete gestacional por Carpenter e Coustan (dois ou mais valores da curva alterados de acordo com a classificação de Carpenter e Coustan ${ }^{6}$ ) e diabete gestacional pelos critérios do NDDG (dois ou mais valores da curva alterados de acordo com a classificação de O'Sullivan e Mahan, modificada pelo NDDG) ${ }^{5}$, podendo uma paciente ser classificada em mais de um grupo.

Realizou-se a comparação desses grupos em relação às características das pacientes (idade, paridade, história de macrossômicos e natimortos, antecedentes familiares de diabete) e aos resultados da gestação atual (pré-eclâmpsia, incidência de operação cesariana, incidência de recémnascidos grandes para a idade gestacional (GIG), natimortalidade e média de peso ao nascer). Para diagnóstico de pré-eclâmpsia, consideraram-se os critérios diagnósticos descritos pelo "National High Blood Pressure Working Education Report"11, ao passo que recém-nascidos GIG foram considerados aqueles acima do percentil 90 para a idade gestacional, conforme a curva de Batagglia e Lubchenco ${ }^{12}$. Definiu-se macrossomia como a história de recém-nascido anterior com peso igual ou superior a $4.000 \mathrm{~g}$.

A coleta da curva glicêmica após a ingestão de $100 \mathrm{~g}$ de glicose seguiu a rotina do serviço, descrita a seguir. As pacientes compareceram ao laboratório, em jejum de pelo menos oito horas, para coleta da glicemia de jejum, recebendo logo após a coleta solução de 100 gramas de glicose anidra, sendo então coletadas amostras de sangue após uma, duas e três horas. O sangue coletado foi colocado em um tubo estéril com anticoagulante da marca Doles, recomendado para dosagem de glicose. Esse anticoagulante é composto por uma solução de EDTA, fluoreto de potássio e sódio. O sangue foi processado para dosagem até, no máximo, três horas após a coleta.

Determinou-se a glicemia plasmática utilizando-se o método enzimático para cada um dos pontos da curva (jejum, uma hora, duas horas e três horas). Essa determinação foi realizada pelos técnicos do laboratório, utilizando-se o kit da marca Doles Glucox 500, baseando-se no método da glicose oxidase. Todas as dosagens anormais foram repetidas.

No período do estudo, foram realizadas no CAM-IMIP trezentas e oitenta e sete curvas glicêmicas. Destas, foram incluídas duzentas e dez pacientes. Entre as pacientes excluídas, noventa e sete foram por parto fora do IMIP, sessenta e quatro por não se encontrarem os prontuários, nove por terem sido realizadas em pacientes não- gestantes e cinco por gestações gemelares. Entre as pacientes incluídas no estudo, os resultados perinatais completos foram obtidos em 133, mas todas tinham registro de peso do recém-nascido.

A análise dos dados foi feita utilizando-se o programa de domínio público Epi-Info 2000. Calculou-se a prevalência de diabete gestacional de acordo com os valores diagnósticos utilizados no CAM-IMIP (Carpenter e Coustan), pelas outras curvas analisadas e a prevalência de hiperglicemia leve. Para comparação dos grupos em relação às características maternas e perinatais, utilizaram-se o teste $\chi^{2}$ de associação para as variáveis categóricas e análise de variância para as variáveis quantitativas. Adotou-se o nivel de significância de $5 \%$.

\section{Resultados}

A amostra foi constituída de 210 pacientes que, de acordo com os niveis glicêmicos encontrados no TOTG, foram enquadradas em um dos grupos definidos nesse estudo. A prevalência de diabete segundo Bertini foi de $48,1 \%$, de acordo com os critérios de Carpenter e Coustan de 18,1\% e 9,0\% segundo o NDDG. Encontramos a prevalência de $10,5 \%$ de pacientes com hiperglicemia leve (Tabela 1).

Tabela 1 - Prevalência de diabetes gestacional (DG) de acordo com o critério diagnóstico utilizado.

\begin{tabular}{lcc}
\hline Critério diagnóstico & $\mathbf{n}$ & $\%$ \\
\hline Controles & 98 & 46,7 \\
Hiperglicemia leve & 22 & 10,5 \\
DG - Carpenter e Coustan & 38 & 18,1 \\
DG - Bertini & 101 & 48,1 \\
DG - NDDG & 19 & 9,0
\end{tabular}

Obs.: uma paciente pode estar incluída em mais de uma categoria

Analisando-se as características das pacientes estudadas, verificou-se que a média de idade variou significativamente entre as gestantes consideradas diabéticas por qualquer parâmetro (Bertini $=28,4$ anos; Carpenter e Coustan $=29,9$ anos; NDDG $=31$ anos) e os controles (26 anos). Não houve diferença significativa da idade entre controles e hiperglicêmicas leves ( 26,7 anos). A mediana de paridade foi de um para todos os grupos. A freqüência de antecedentes familiares de diabete e história de natimortos ou macrossômicos não apresentou diferença significativa entre os grupos (Tabela 2). 
Tabela 2 - Características das pacientes do estudo de acordo com o critério diagnóstico adotado.

\begin{tabular}{|c|c|c|c|c|c|c|}
\hline Grupo & Controles & HG leve & DG-B * & DG-CCE & DG-NDDG \# & $p$ \\
\hline \multirow[t]{2}{*}{ Idade (média \pm DP) } & 26,00 & 26,72 & 28,40 & 29,97 & 31,05 & 0,0006 \\
\hline & $(5,51)$ & $(6,13)$ & $(6,77)$ & $(6,43)$ & $(6,72)$ & \\
\hline \multirow[t]{2}{*}{ Macrossômicos anteriores (n/\%) } & 17 & 1 & 17 & 9 & 5 & 0,3 \\
\hline & $(16,2)$ & $(4,5)$ & $(16,8)$ & $(23,7)$ & $(26,3)$ & \\
\hline Natimortos anteriores $(\mathrm{n} / \%)$ & $(5,8)$ & $(9,1)$ & $(5,9)$ & $(0,0)$ & $(0,0)$ & \\
\hline \multirow[t]{2}{*}{ Antecedentes familiares (n/\%) } & 46 & 12 & 49 & 21 & 13 & 0,3 \\
\hline & $(43,8)$ & $(54,5)$ & $(49,0)$ & $(55,3)$ & $(68,4)$ & \\
\hline
\end{tabular}

* DG-B = Diabetes gestacional pelos critérios de Bertini

$£$ DG-CC = Diabetes gestacional pelos critérios de Carpenter e Coustan

\# DG-NDDG = Diabetes gestacional pelos critérios do NDDG

$\mathrm{HG}=$ Hiperglicemia

A freqüência de pré-eclâmpsia foi significativamente maior entre as pacientes com hiperglicemia leve $(50 \%)$ e diabete gestacional por Carpenter e Coustan (50\%) em relação aos controles $(28,6 \%)$. Observou-se tendência a maior freqüência de pré-eclâmpsia em pacientes com diabete pelos critérios de Bertini (40\%), porém não houve diferença nessa freqüência entre controles e gestantes classificadas como diabéticas pelos critérios do NDDG $(36,8 \%)$ (Tabela 3$)$. Em relação à operação cesariana, não houve diferença estatisticamente significativa entre as cinco classes $(p=0,69)$ (Tabela 3$)$.

Tabela 3 - Resultados maternos de acordo com o critério diagnóstico adotado.

\begin{tabular}{lcccccc}
\hline Resultados & \multicolumn{2}{c}{ Pré-eclâmpsia } & \multicolumn{4}{c}{ Cesariana } \\
Grupos & $\mathbf{n}$ & $\%$ & $\mathbf{p}$ & $\mathbf{n}$ & $\%$ & $\mathbf{p}$ \\
\hline Controles & 30 & 28,6 & & 54 & 51,9 & \\
HG leve & 11 & 50,0 & 0,05 & 12 & 57,1 & 0,66 \\
DG-B & 40 & 40,0 & 0,084 & 49 & 49,5 & 0,79 \\
DG-CC & 19 & 50,0 & 0,01 & 21 & 55,3 & 0,72 \\
DG-NDDG & 7 & 36,8 & 0,46 & 7 & 36,8 & 0,22 \\
\hline
\end{tabular}

Fonte: Pesquisa do IMIP.

* DG-B = Diabetes gestacional pelos critérios de Bertini

$£$ DG-CC = Diabetes gestacional pelos critérios de Carpenter e Coustan

\# DG-NDDG = Diabetes gestacional pelos critérios do NDDG

$H G=$ Hiperglicemia

Não se evidenciou diferença significativa entre as classes e m relação à freqüência de GIG $(p=0,69)$, resultado este que persistiu quando a análise foi feita classe a classe, em relação aos controles. No entanto, verificou-se uma tendência a maior freqüência de GIG entre as pacientes com hiperglicemia leve $(p=0,09)$ (Tabela 4).
Avaliou-se a freqüência de natimortos em cada uma das classes, não se encontrando diferença significativa entre os grupos $(p=0,97)$, bem como entre cada grupo e os controles (Tabela 4). Em relação ao peso ao nascer, não se evidenciou diferença estatisticamente significativa entre os grupos ( $p=0,5)$, porém, ao se analisarem as classes separadamente, observouse tendência à significância estatística quando as pacientes diabéticas por Carpenter e Coustan foram comparadas com os controles $(\mathrm{p}=0,07)$ (Tabela 4).

\section{Discussão}

A prevalência de DG e hiperglicemia leve encontrada no presente estudo pode ser considerada elevada em relação à população geral. Deve-se lembrar, entretanto, que todas as pacientes incluídas na amostra tinham, por alguma indicação, realizado um TOTG, caracterizando desta forma uma população selecionada e de alto risco para o evento. Resultados semelhantes foram descritos anteriormente, observando-se aproximadamente $20 \%$ de resultados positivos ${ }^{13}$.

Em relação às características das pacientes, encontramos aumento progressivo da idade à medida que aumentavam os níveis glicêmicos. Na verdade, diversos autores demonstraram que pacientes com DG tendem a ser mais velhas ${ }^{14}$, podendo este fato ser explicado pelo aumento da incidência de intolerância aos carboidratos com a idade. 
Tabela 4 - Resultados perinatais de acordo com o critério diagnóstico adotado.

\begin{tabular}{|c|c|c|c|c|c|c|c|c|c|}
\hline \multirow{2}{*}{$\begin{array}{l}\text { Resultados } \\
\text { Grupos }\end{array}$} & \multicolumn{2}{|c|}{ GIG } & \multirow[b]{2}{*}{$p$} & \multicolumn{2}{|c|}{ Natimortos } & \multirow[b]{2}{*}{$p$} & \multicolumn{2}{|c|}{ Peso ao nascer } & \multirow[b]{2}{*}{$p$} \\
\hline & $\mathrm{n}$ & $\%$ & & $\mathbf{n}$ & $\%$ & & g & DP & \\
\hline Controles & 18 & 17,8 & & 3 & 3,1 & & 3.096 & 643 & \\
\hline DG-B & 22 & 22,2 & 0,45 & 3 & 3,3 & 0,92 & 3.190 & 749 & 0,33 \\
\hline DG-CC & 10 & 26,3 & 0,26 & 1 & 2,9 & 0,95 & 3.328 & 781 & 0,07 \\
\hline
\end{tabular}

* DG-B = Diabetes gestacional pelos critérios de Bertini

$£$ DG-CC = Diabetes gestacional pelos critérios de Carpenter e Coustan

\# DG-NDDG = Diabetes gestacional pelos critérios do NDDG

$\mathrm{HG}=$ Hiperglicemia

Em relação à paridade, história de macrossômicos ou natimortos e história familiar de diabete, não encontramos associação com o critério diagnóstico utilizado. Esse achado difere das conclusões de diversos outros estudos, porquanto a literatura pertinente refere significativa associação entre a presença de DG e obesidade, história familiar de diabete melito, tabagismo, antecedentes pessoais de DG, macrossômicos e natimortos ${ }^{14}$. Tal achado deve-se, possivelmente, ao fato de as pacientes estudadas possuírem uma maior possibilidade de ter fatores de risco, porque a presença destes é um das indicações para o exame de rastreamento a que todas se submeteram.

Deve-se destacar a evidência de uma freqüência aumentada de operação cesariana em relação à esperada para a população atendida no serviço, observando-se tal fato em todos os grupos, inclusive os controles. Todavia, estes não diferiram entre si. Essa freqüência elevada não pode ser comparada com números gerais, porque nossa amostra já apresenta, per se, um viés de seleção importante, considerando que o IMIP atende preferencialmente gestantes de alto risco. Como os controles, na verdade, eram pacientes que tiveram alguma indicação para rastreamento e realizaram posteriormente um exame diagnóstico para diabete, estas pacientes constituem, na verdade, grupo com risco aumentado para cesariana.

Verificou-se significativo aumento da freqüência de pré-eclâmpsia entre as pacientes com hiperglicemia leve e DG pelos critérios de Carpenter e Coustan (50\%) em relação aos controles $(28,6 \%)(p=0,01)$. Apesar de ter sido verificada tendência a maior freqüência de pré-eclâmpsia entre os casos com DG pelos critérios de Bertini $(40 \%)$, essa diferença não chegou a ser estatisticamente significativa $(p=0,08)$. Naturalmente, $o$ tamanho da amostra pode ter sido insuficiente para evidenciar essa diferença. É importante assinalar que mesmo entre os controles a freqüência de pré-eclâmpsia foi bastante elevada (28\%) em relação à freqüência observada no serviço (em torno de $10 \%$ ), o que também pode ser explicado pelo fato de a população estudada ser constituída de gestantes que já tinham passado por uma triagem inicial e por isso poderiam ter maior risco para o evento, o que representaria um viés de seleção. A relação entre pré-eclâmpsia e diabete gestacional tem sido descrita na literatura, tendo sido demonstrada em análise secundária do grande estudo multicêntrico CPEP ("Calcium for Preeclampsia Prevention Study Group”), em 1998. Nesse estudo, mulheres com DG mostraram risco aumentado para distúrbios hipertensivos durante a gravidez, mesmo depois do ajuste para variáveis confundidoras. Os autores concluíram que o achado confirmava a hipótese de que a resistência à insulina pode ter um papel na patogênese das síndromes hipertensivas na gestação ${ }^{15}$.

Achado bastante relevante, em nosso estudo, foi a ausência de diferença na freqüência de natimortos, macrossômicos e de GIG, bem como entre as médias de peso ao nascer, em todos os grupos com diagnóstico de diabete pelos variados critérios e os controles. Esses resultados condizem com os publicados por Pennison e Egerman ${ }^{16}$, que não puderam confirmar os benefícios da utilização dos critérios de Carpenter e Coustan, posto que estes utilizam níveis mais baixos de glicemia e são portanto mais inclusivos, em relação àqueles preconizados pelo NDDG. Concluíram não serem claros os benefícios do uso de critérios mais inclusivos para o diagnóstico de DG, reforçando assim a opinião de outros autores ${ }^{17}$.

Por outro lado, há autores que acreditam que o uso dos valores do NDDG levaria a subdiagnóstico e que uma fração não desprezivel de pacientes ficaria sem tratamento ${ }^{18}$. As possiveis repercussões desse subdiagnóstico, no entanto, não são conhecidas, e se o prognóstico perinatal é pior nos casos não diagnosticados por critérios mais rígidos, isso não foi ainda demonstrado na literatura.

Em relação às pacientes com apenas um va- 
lor do TOTG alterado, diversos estudos demonstram que essas teriam pior resultado perinatal. $\mathrm{O}$ estudo de Langer et $a{ }^{19}$ foi o primeiro de uma série começando a questionar o dogma da definição do DG com base em dois valores alterados, demonstrando que os resultados perinatais de pacientes com apenas um valor alterado eram semelhantes aos de pacientes com diagnóstico de DG. Vambergue et al. ${ }^{9}$ denominaram essas pacientes como portadoras de hiperglicemia leve, apresentando uma taxa de macrossômicos quase duas vezes maior. Em nossos resultados, apesar de existir tendência ao aumento da freqüência de GIG, operação cesariana e das médias de peso ao nascer, essa diferença não foi significativa, mas talvez pudesse ter sido encontrada com um maior tamanho amostral.

Outro ponto a ser destacado diz respeito ao que representa para a gestante o rótulo do diagnóstico de diabete. Jarret ${ }^{20}$, em 1993, destacou que, sem dúvida, a incidência de operação cesariana aumenta quando o médico se "conscientiza" do diagnóstico e Lucas et al. ${ }^{21}$, em 1993, chamaram a cesariana de "morbidade associada ao diagnóstico". No artigo de Jarret, questiona-se até mesmo se o DG chega a ser uma entidade clínica, apontando-se que seu rastreio pode estar causando mais malefícios que benefícios.

Por outro lado, o risco aumentado de desenvolver diabete melito futuro após diagnóstico de DG também poderia ser modificado por alterações dietéticas e comportamentais iniciadas durante a gestação. Feig et al. ${ }^{22}$ em 1998, publicaram estudo avaliando a visão do próprio status de saúde por mulheres 3 a 5 anos após o diagnóstico de DG, comparando-as com controles. Observou que o diagnóstico efetuado na gestação estava associado a mudanças na forma com que essas pacientes viam sua própria saúde e a saúde da criança nascida da gestação afetada, o que poderia levá-las a adotar medidas para tornar sua vida mais saudável.

Danilenko-Dixon et $\mathrm{al}^{14}$., avaliando o impacto da aplicação dos critérios de Carpenter e Coustan, afirmam que, apesar de trinta anos de estudo, a falta de consenso em relação ao diabete gestacional permanece em praticamente todos os aspectos clínicos (necessidade de rastrear, critérios diagnósticos e tratamento) e até mesmo questionam o DG como diagnóstico válido.

De forma semelhante, Buchanan \& $\mathrm{Kjos}^{23}$, em seu artigo "Diabetes gestacional: risco ou mito?", questionam que, se por um lado, ignorar a hiperglicemia na gestação pode acarretar inevitável aumento da freqüência de morbidades associadas, por outro, a detecção e tratamento excessivamente agressivos levarão a "supertratamento" de algumas pacientes ${ }^{23}$.
O presente estudo apresenta uma visão crítica em relação aos métodos diagnósticos utilizados atualmente para o DG. Para responder a essas questões, novos estudos prospectivos devem ser realizados, envolvendo um maior número de casos e controlando de forma eficiente os fatores de confusão.

\section{ABSTRACT}

Purpose: to analyze the perinatal results of patients submitted to a $100 \mathrm{~g}$ oral glucose tolerance test (OGTT) during prenatal care at the Instituto Materno-Infantil de Pernambuco (IMIP), according to three different criteria.

Methods: a cross-sectional study was conducted involving 210 pregnant patients attended at the IMIP, who were tested by a $100 \mathrm{~g}$ OGTT and had a singleton, topic pregnancy, without history of diabetes or glucose intolerance before pregnancy, and who delivered at the IMIP. The patients were classified into one of the following categories according to the levels found by OGTT: controls, mild hyperglycemia, Bertini's group, Carpenter's group and the National Diabetes Data Group (NDDG). These classes were then compared and association between the categories and preeclampsia, large for gestational age (LGA) newborns, rate of cesarean delivery, stillbirth, and mean birth weight was investigated. Results: the frequency of gestational diabetes was 48.1, 18.1, and $9 \%$ according to Bertini's, Carpenter and Coustan's and NDDG criteria, respectively, and mild hyperglycemia was present in $10.5 \%$. Age of patients increased with a higher degree of carbohydrate intolerance. The groups did not differ regarding frequency of LGA, C-section, stillbirths, and birth weight. There was an increased frequency of preeclampsia among women with hyperglycemia and gestational diabetes according to Carpenter and Coustan's criteria.

Conclusions: prevalence of gestational diabetes varied between 9 and 48\% according to the different criteria, but maternal and perinatal results did not differ significantly among the groups. Strict diagnostic criteria can determine overdiagnosis without improvement of perinatal outcome.

KEY WORDS: Gestational diabetes. Hypertension. Fetal death. Oral glucose tolerance test.

\section{Referências}

1. Report of the Expert Committee on the Diagnosis and Classification of Diabetes Mellitus. Diabetes Care 1997; 20:1183-97.

2. Santos LC, Carvalho MMRA, Porto AM, Guimarães V. Obstetrícia (IMIP): diagnóstico e tratamento. Rio de Janeiro: MEDSI; 1997. p.375-90. 
3. Solomon CG, Willet WC, Rich-Edwards J, et al. Variability in diagnostic evaluation and criteria for gestational diabetes. Diabetes Care 1996; 19:12-6.

4. O'Sullivan JB, Mahan CM. Criteria for the oral glucose tolerance test in pregnancy. Diabetes 1964; 13:278-85.

5. National Diabetes Data Group. Classification and diagnosis of diabetes mellitus and other categories of glucose intolerance. Diabetes 1979; 28:1039-57.

6. Carpenter MW, Coustan DR. Criteria for screening tests for gestational diabetes. Am J Obstet Gynecol 1982; 144:768-73.

7. Naylor CD. Diagnosing gestational diabetes mellitus. Is the gold standard valid? Diabetes Care 1989; 12:565-72.

8. Metzger BE, Coustan DR. Summary and recommendations of the Fourth International Workshop-Conference on gestational diabetes mellitus. Diabetes Care 1998; 21 (Suppl 2): B161-7.

9. Vambergue A, Nuttens MC, Verier-Mine O, Dognin C, Cappoen JP, Fontaine P. Is mild gestational hyperglycaemia associated with maternal and neonatal complications? The Diagest Study. Diabet Med 2000; 17:203-8.

10. Oliveira AM, Camano L. Rastreamento e diagnóstico do diabetes gestacional: considerações preliminares. J Bras Ginecol 1983; 93:263-72.

11. Report of the National High Blood Pressure Education Program Working Group on High Blood Pressure in Pregnancy. Am J Obstet Gynecol 2000; 183:S1-S22.

12.Battaglia FC, Lubchenco LO. A practical classification of newborn infants by weight and gestational age. $\mathrm{J}$ Pediatr 1967; 71:159-63.

13.Rudge MVC, Calderon IMP, Ramos MD, Suetake H, Peraçoli JC. Investigação diagnóstica do diabetes na gestação. Rev Bras Ginecol Obstet 1996; 18:21-8.
14.Danilenko-Dixon DR, Van Winter JT, Nelson RL, Ogburn PL Jr. Universal versus selective gestational diabetes screening: application of 1997 American Diabetes Association recommendations. Am J Obstet Gynecol 1999; 181:798-802.

15.Joffe GM, Esterlitz JR, Levine RJ, et al. The relationship between abnormal glucose tolerance and hypertensive disorders of pregnancy in healthy nulliparous women. Am J Obstet Gynecol 1998; 179:1032-7.

16.Pennison EH, Egerman RS. Perinatal outcomes in gestational diabetes: a comparison of criteria for diagnosis. Am J Obstet Gynecol 2001; 184:1118-21.

17.Schwartz ML, Ray WN, Lubarsky SL. The diagnosis and classification of gestational diabetes mellitus: is it time to change our tune? Am J Obstet Gynecol 1999; 180:1560-71.

18.Magee MS, Walden CE, Benedetti TJ, Knopp RH. Influence of diagnostic criteria on the incidence of gestational diabetes and perinatal morbidity. JAMA 1993; 269:609-15.

19.Langer O, Brustman L, Anyaegbunam A, Mazze R. The significance of one abnormal glucose tolerance test value on adverse outcome in pregnancy. Am J Obstet Gynecol 1987; 157:758-63.

20.Jarrett RJ. Gestational diabetes: a non-entity? BMJ 1993; 306:37-8.

21.Lucas MJ, Lowe TW, Bowe L, McIntire DD. Class A1 gestational diabetes: a meaningful diagnosis? Obstet Gynecol 1993; 82:260-5.

22.Feig DS, Chen E, Naylor CD. Self-perceived health status of women three to five years after the diagnosis of gestational diabetes: a survey of cases and matched controls. Am J Obstet Gynecol 1998; 178:386-93.

23.Buchanan TA, Kjos SL. Gestational diabetes: risk or myth? J Clin Endocrinol Metab 1999; 84:1854-7. 